\title{
Behavior of taxi customers in hailing vacant taxis: a nested logit model for policy analysis
}

\author{
Ryan C.P. Wong, W.Y. Szeto* and S.C. Wong \\ Department of Civil Engineering, The University of Hong Kong, Hong Kong \\ *E-mail: ceszeto@hku.hk \\ Phone: (852) 28578552 \\ Fax: (852) 25595337
}

\begin{abstract}
SUMMARY
This study models and examines the taxi customers' preferences for hailing vacant taxis on streets. A stated preference survey was conducted to randomly select and interview 1,242 taxi customers at taxi stands and pedestrians on streets, who had experiences of taking taxis recently, about their choices under different given hypothetical scenarios. In total, 4,968 observations were collected and used for developing the discrete choice models for the analysis. To account for the potential correlations among alternatives, two nested logit models are developed, calibrated, and compared with a standard multinomial logit model in the investigation. The results of likelihood ratio test demonstrate that one of the developed nested logit models is better than the standard multinomial logit model to describe the search behavior of taxi customers. The model results also show that the walking time to and the waiting time at the location for hailing taxis, the extra travel time to the destination because of local circulation for finding a way from the pickup location heading to a passenger's destination, as well as the taxi customers' perceptions for walking to and waiting at taxi stands were found as significant factors to influence their decisions. In addition, the results of market segmentation analysis illustrate the variations in taxi-search strategies of taxi customers in different districts and regions. Some policy implications on introducing more taxi stands and improving the utilization rates of taxi stands are also discussed. We believe that the proposed models, findings, and discussion are useful for developing micro-simulation models to evaluate the performance of road traffic networks with taxi services and developing simulation-based optimization models to answer policy questions related to taxi services.
\end{abstract}

KEY WORDS: urban taxis; taxi hailing behavior; taxi stand; stated preference survey; nested logit model

\section{INTRODUCTION}

Taxis provide door-to-door transportation services. It can be used as a complement mode to existing public transit services [1, 2]. However, they consume road space and worsen traffic congestion and air pollution problems, especially when they circulate on roads in search of customers as in many parts of the world. Moreover, there is always a mismatch between demand and supply of taxis over time and space. As a result, the taxi system is often inefficient and the customers do not satisfy with the service. Furthermore, poorly designed taxi bays have insufficient capacity, causing long taxi queues extending onto nearby roads and obstructing local traffic. To tackle these problems, advanced technologies [3-8] and taxi 
regulation policies [9-13] are often introduced. However, the taxi regulation policy studies have been developed based on an idealized market and conventional economic analysis in which the spatial structure of the market is ignored.

In an attempt to capture the spatial structure of the market, Yang and Wong [14] developed a model to determine the taxi movements on a given road network for a given customer origin-destination (OD) demand pattern. Later, the model was improved in numerous studies [15-21] to capture congestion effects, multiple user classes, multiple taxi modes, customer hierarchical modal choice, day-to-day learning processes, stochastic travel time, and local customer-search behavior. Solution algorithms were also developed by Wong and Yang [22] and Wong et al. [23, 24] for solving the improved models. In these existing taxi network models, multinomial logit (MNL) models were used to describe the search behavior of vacant taxi drivers. However, the logit model had not been calibrated and validated.

To calibrate and validate the MNL models, Sirisoma et al. [25] performed a stated preference survey. Wong et al. [26] calibrated and validated the logit models based on global positioning system (GPS) data from 460 urban taxis to predict the drivers' strategic zonal choice for searching for customers during both peak and off-peak periods. Szeto et al. [27] further extended the consideration to every hour in a day, and Wong et al. [28] extended [26] by adopting the sequential logit approach to modeling sequential customer-search decisions of vacant taxi drivers on finding customers at intermediate zones while heading to their designated zones. However, these behavioral studies of vacant taxi drivers focused on their zonal choices in search of customers. Their models cannot be used for developing microsimulation-based models and simulation-based optimization models for depicting traffic flow including taxi flow on local streets, managing local traffic, evaluating the utilization rates of taxi stands and the capacities of taxi bays, and determining the optimal number and locations of taxi stands in each zone with the consideration of utilization rates because some choice decisions of vacant taxi drivers, such as traveling to a taxi stand, are not modeled in the zone choice model. More vacant taxi driver decisions are also needed to model for these applications.

To address this issue, Kitamura and Yoshii [29] adopted GPS data to develop a singlelevel discrete choice model to model their preferences for traveling towards taxi stands. Wong et al. [30] conducted a questionnaire survey to 258 urban taxi drivers and developed a sequential logit model to depict their customer-search preferences for traveling towards taxi stands. This study determined the factors that affect taxi drivers' customer-search decisions and discussed potential taxi policy implications on enhancing the utilization rates of taxi stands and on introducing additional taxi stands in different taxi shifts and service regions. They also pointed out that taxi drivers' decisions are not only simply based on the travel distance and the congestion level on the way of searching, but also based on the number of passengers waiting at taxi stands. However, as in most of the literature, they ignored the movements of taxi customers to get taxis, which in fact determine the origins of the occupied taxi trips and affect the utilization of taxi stands. In particular, more people waiting at the roadside for hailing taxis imply lower utilization rates of nearby taxi stands and more taxis cruising on roads instead on staying at taxi stands, leading to more an inefficient taxi market and a more congested and polluted environment. It is therefore extraordinarily important to consider taxi-customer movements when developing simulation or simulation-optimization models to improve the taxi market and environment.

To our best knowledge, very few studies consider the behavior of hailing taxis, except that Wong et al. [31] proposed a bilateral model to determine both the customers and vacant taxi movements on the road networks. They modeled the location choice of taxi customers of 
waiting vacant taxis according to perceived generalized travel cost minimization, where the generalized cost is a function of the walking time to and the waiting time at the designated location for hailing taxis and the in-vehicle travel time from that location to the destination. Later, the model was further improved in [32-34] to consider the search frictions between vacant taxis and taxi customers, analyze the model equilibrium properties, and deal with the congestion effect. However, the assumptions of these models have not been verified and these models ignore the following. First, taxi customers may have a bias on waiting at the roadside. Second, the waiting location affects the in-vehicle travel time and hence the fare and generalized travel cost.

To address the above issues, this paper proposes and calibrates discrete choice models for depicting the preferences of taxi customers for hailing vacant taxis on streets. To calibrate the model for future use in simulation or simulation-optimization studies, we conducted a stated preference (SP) questionnaire survey. We randomly selected and interviewed 1,242 taxi customers at taxi stands and pedestrians on streets who had experiences of taking taxis recently. They were invited to report their choices under four hypothetical games and hence we obtained 4,968 observations for model calibration. We adopt the standard MNL modeling approach at first to modeling the preference of taxi customers of hailing vacant taxis on streets. In addition, to account for the potential correlations among the alternatives in the hypothetical games, nested logit (NL) models are also calibrated in the investigation. The NL modeling approach has been commonly used to resolve the independence of irrelevant alternatives (IIA) assumption of the MNL model and analyze the alternative similarity [3539]. This study recommends the best model structure based on the results of the likelihood ratio test. This study further investigates whether the taxi customers' decisions change across different market segments in terms of districts, (time) periods, and regions in search of vacant taxis, by developing individual models and carrying out Watson and Westin pooling test [40]. The paper also discusses potential taxi policy implications on enhancing the utilization rates of taxi stands and on introducing additional taxi stands in different districts and regions. The main contributions of this paper are as follows. (1) Proposing and calibrating discrete choice models to depict the taxi-search behavior of taxi customers; this study should be one of the earliest papers, if not the first paper, to propose and calibrate a taxi-search model using real data; (2) Determining the factors that affect the choices of taxi customers on traveling towards the nearest taxi stand to get a taxi; (3) Discussing the potential implications of implementing taxi policies, such as introducing more taxi stands in different service regions, and implementing policies to increase the utilization rates of taxi stands, and; (4) Providing a sub-model for developing micro-simulation models to evaluate the performance of road traffic networks with taxi services and developing simulation-based optimization models to answer policy questions related to taxi services.

The remainder of this paper proceeds as follows. Section 2 describes the data collection method, the demographical distribution of interviewed taxi customers, and their frequencies of taking taxis and preferences for waiting at taxi stands. Section 3 presents the model formulations of the MNL and NL models, the model selection criteria, and the methodology of market segmentation analysis. Section 4 discusses the results and policy implications. Finally, Section 5 concludes the paper and suggests research directions for future study. 


\section{DATA}

\subsection{Data collection}

At present, 18,138 taxis are operating in Hong Kong territory. They include 15,250 urban taxis, 2,838 New Territories taxis, and 50 Lantau taxis, serving about 1 million occupied trips a day. Urban taxis operate at most areas in Hong Kong (Hong Kong Island, Kowloon Peninsula, and the New Territories); New Territories taxis mainly operate in the north-eastern part and north-western part of New Territories; and Lantau taxis are permitted to operate on Lantau Island and Chep Lap Kok (the airport) only. Figure 1 shows the district map of Hong Kong and the associated permitted operating areas for different types of taxis. The taxi fare structure was the same throughout the day, without surcharges at night or during peak hours. The travel fares of New Territories and Lantau taxis were charged similarly, but with lower rates.

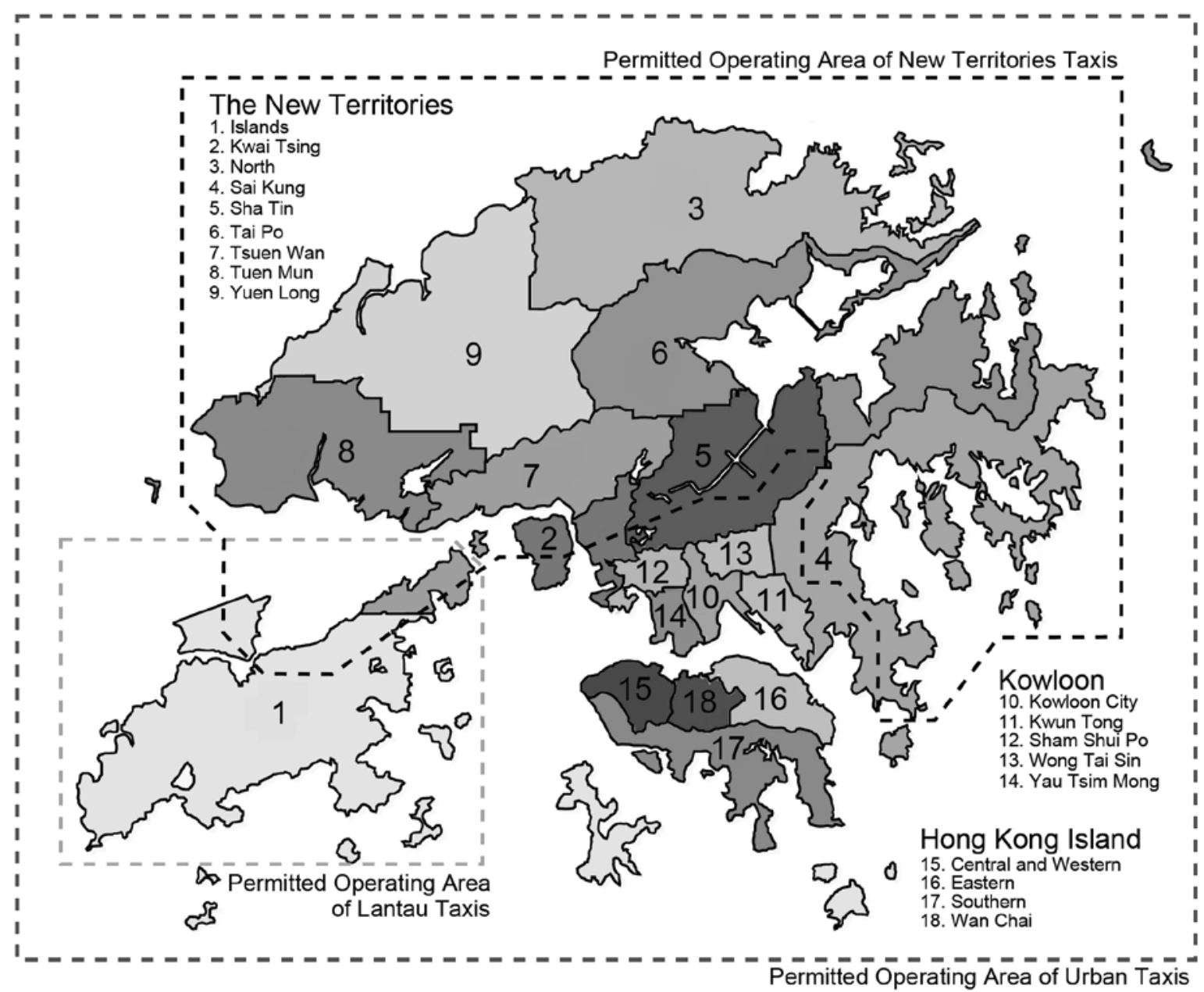

Figure 1. District map and permitted operating areas of taxis

The Hong Kong Transport Department set up 468 roadside taxi stands in total; 79 are in Hong Kong Island; 107 are in Kowloon Peninsula; 252 are in New Territories, and remaining 30 are in Lantau Island. Most of them are ordinary on-street taxi stands specifically providing services for one type (sometimes two types) of taxis, and less than $10 \%$ of them are cross- 
harbor taxi stands that serve the taxi customers with their destinations at the opposite side of Victoria Harbor. Amongst all these on-street taxi stands, a few of them are operated in either day-time or night-time only. This arrangement prevents further worsening traffic congestion at urban areas during peak hours due to one traffic lane occupied by taxis queuing in front of an on-street taxi stand and caters the variations in passenger demand with spatial and temporal considerations.

The SP questionnaire survey was conducted from January to March 2013 in the daytime and at nighttime. The face-to-face interview questionnaire surveys were taken place in selected residential and commercial districts in Hong Kong Island, Kowloon Peninsula, and the New Territories. We randomly selected and interviewed taxi customers who were waiting at taxi stands and pedestrians on streets who had experiences of taking taxis recently. In the case when respondents did not have sufficient time to finish the questionnaires during the face-to-face interview, they were invited to complete questionnaires by themselves and then mailed back the completed questionnaires to us by using an attached return envelope. We collected 1,242 responses in total, where about two-third of them were collected during faceto-face interviews on site and the rest were collected by mail. The overall response rate was about 23\% (calculated as the number of received questionnaires divided by the number of people we approached for interview).

\subsection{Demographical distribution and taxi-search pattern of interviewed taxi customers}

The questionnaire has two sections. The first section collected the demographics information from interviewed taxi customers such as gender, age, occupation status, education level, monthly income, as well as their most frequent periods and regions of taking taxis in their experiences. The summary of the demographic distribution is shown in Figure 2. 


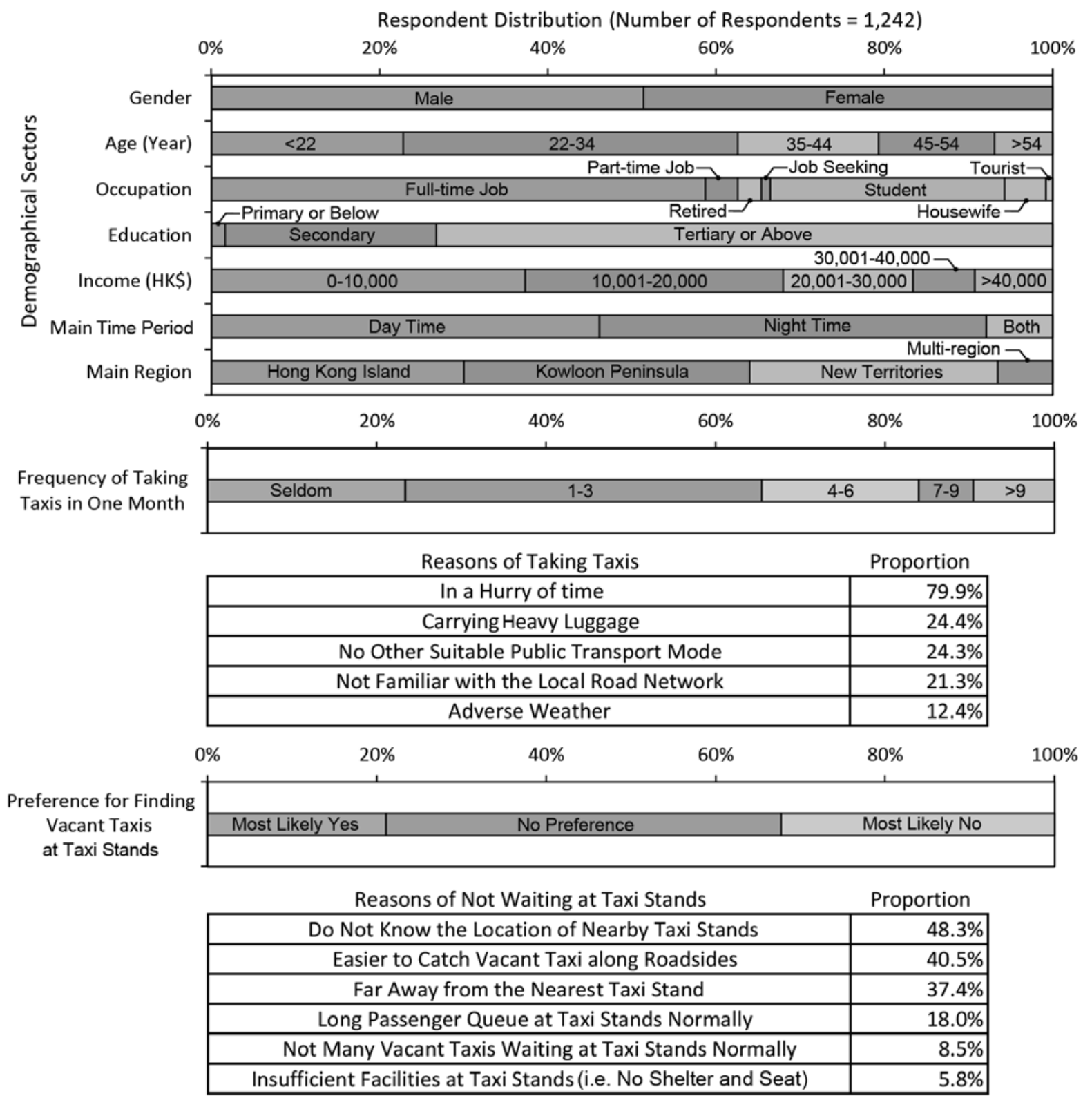

Figure 2. Interviewed taxi passengers' demographical distribution and their frequencies of taking taxis and preferences for finding vacant taxis at taxi stands

It is noticed that about half of the interviewed taxi customers (51\%) were male. Around $80 \%$ of them were aged below 45 and concentrated at the age group of 22-34. For the occupation status of the interviewees, $63 \%$ of them had either a full-time or a part-time job; $28 \%$ of them were students, and remaining $10 \%$ were either retired, seeking for job, housewives, or tourists. The majority of the interviewed taxi customers reported that they were educated up to tertiary level or above. We attempted to categorize the income levels into five groups. It is noticed that more than $60 \%$ of people taking taxis had incomes more than HK\$10,000 per month.

To analyze the spatial-temporal distribution of passenger demands, the most frequent periods and regions of taking taxis in their experience were questioned. The results illustrate that only about $8 \%$ of the respondents did not have a fixed period of taking taxis, and the rest were classified into two groups, namely day time (05:00-17:00) and night time (17:00-05:00) periods. The identified periods are actually the operating hours of taxi drivers in different taxi shifts. This setting facilitates us to compare our taxi customer model with a taxi driver model 
in the later stage. The most frequent regions of taking taxis got a similar phenomenon that about $7 \%$ of the respondents took taxis in multi-region normally, and the rest were equally distributed in Hong Kong Island, Kowloon Peninsula, and the New Territories.

Other than the personal particulars of the interviewed taxi customers were obtained, the second section of the questionnaire requested the respondents to report their frequencies of taking taxis and general preferences for finding vacant taxis at taxi stands in order to evaluate the popularity of using taxi stands in Hong Kong.

Figure 2 shows the monthly frequencies and most popular reasons of the interviewed taxi customers taking taxis. It is observed that slightly less than $80 \%$ of the respondents took taxis at least once a month, in particular about $40 \%$ of them traveled by taxis one to three times monthly. The most popular reason of taking taxis was in a hurry of time, with about $80 \%$. The following reasons that presented in the decreasing order of the percentage were carrying heavy luggage, no other public transport mode available to the destinations, not familiar with the local road network nearby, and traveling by taxis because of adverse weather. From the results, we can have the following conclusions: 1) In a hurry of time was chosen as the highest ranked reason, pointing out that time minimization was the most important factor affecting their choices of taking taxis; and 2) Carrying heavy luggage was anticipated to cause taxi customers searching for vacant taxis in a shorter walking distance. It implies that walking distance minimization was also one of the major concerns of taxi customers.

Figure 2 further presents their preferences for finding vacant taxis at taxi stands and the major reasons of taxi customers not waiting at taxi stands. The taxi stands included the ordinary roadside taxi stands, and the taxi waiting points at hotels, shopping malls, major commercial buildings, and residential estates. It is noted that only $20 \%$ of taxi customers most likely wait for taxis at taxi stands. The proportion was obviously lower than that of most likely not going to. The majority of the interviewed taxi customers claimed that they had no particular preference and they would make their decisions subject to the real-time conditions. It was a good indication that if correct transportation policies in prompting taxi customers using taxi stands were implemented, these customers would use taxi stands. For the reasons of the customers not preferring for waiting at taxi stands, most of the respondents expressed that it was because they did not know the location of the nearby taxi stand (not familiar with the local road network). The others considered that it was easier to them to catch vacant taxis along the roadside and the walking distance to the nearest taxi stand was too long. They also had an expectation that a lot of people waited at taxi stands normally but not many vacant taxis went there in search of customers. Only $6 \%$ of the interviewees reported that insufficient facilities (e.g., no shelter and seat) at taxi stands adversely influenced their decisions of hailing vacant taxis there.

\subsection{Decisions of hailing vacant taxis on streets}

In a normal situation when a taxi trip is not well planned in advance and a customer does not call a vacant taxi through telephone order, the customer has three possible choices to search for a vacant taxi on streets: 1) Waiting at the roadside of the current position; 2) Walking to and waiting at the roadside of a nearby main street (a place where normally offers a higher chance to meet a vacant taxi); and 3) Walking to and waiting at a nearby taxi stand. Figure 3 provides a graphical example to show the three possible choices for a taxi passenger to search for a vacant taxi on streets. (C, M, and S represent the current position, the location of a nearby main street, and the location of a taxi stand, respectively.) The taxi customer is anticipated to judge the time consumptions of these options based on his/her experience of hailing vacant taxis at these three locations. Note that the associated walking and waiting 
times are indeed the perceived values of this taxi customer before he/she actually catches a vacant taxi there. Furthermore, it is abnormal that a taxi customer goes for a lengthy walking distance (say, walk across districts) to search for a vacant taxi. Hence, we assume that taxi customers only consider the location within a walkable distance to get taxis.

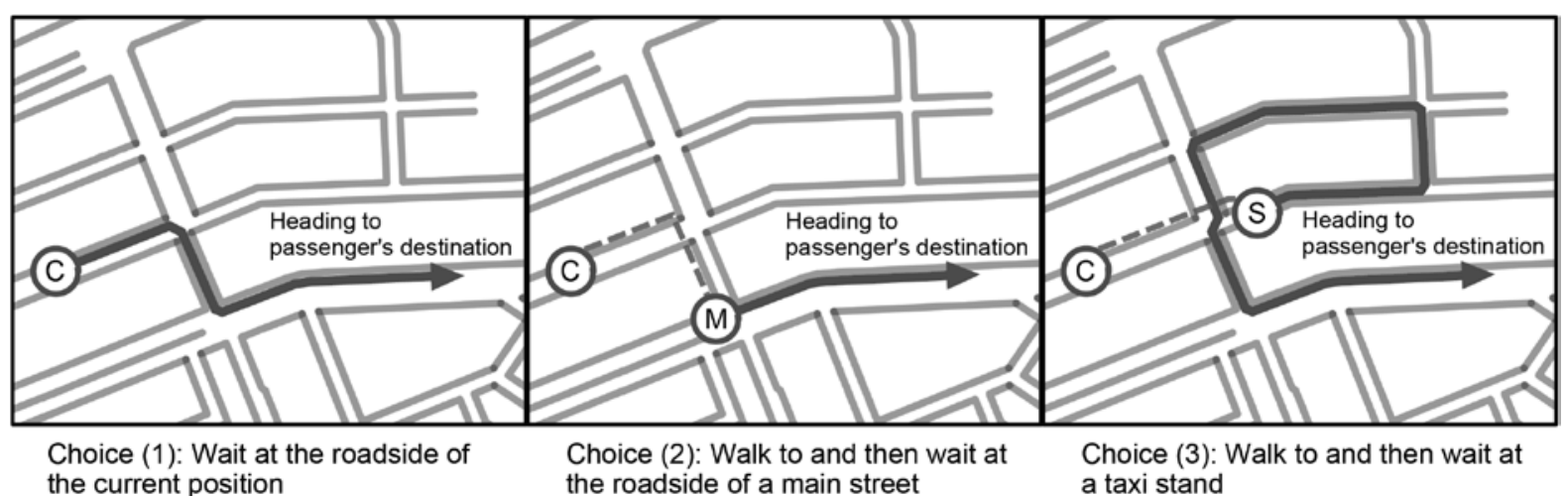

Figure 3 A graphical example for the three possible choices to search for a vacant taxi

Taxi customers made a decision on selecting one of these three choices based on some attributes. Wong et al. [31] suggested that the taxi customers made decisions on finding vacant taxis on streets based on perceived generalized travel cost minimization, where the key components of the generalized travel cost are the walking time to and the waiting time at the designated location for hailing taxis, and the in-vehicle travel time from that location to the destination. The in-vehicle travel time consists of two components: 1) Local circulation time (which is the extra travel time to the destination due to circulation at the local road network after picking up customers to find a way heading to the passenger's destination; depending on the location to start up the occupied trip and the local traffic stream direction, the occupied taxi may need to do the circulation); and 2) Travel time that follows immediately after the local circulation time until this occupied taxi arrives at the destination. As a matter of fact, the latter component should make no difference to taxi customers' decisions of searching for taxis locally, and only the length of time prolonged for local circulation could affect their search options. Therefore, this paper introduces the local best (waiting) point concept for hailing taxis on streets. The traffic stream direction at the local best point is already heading to the customers' destinations. If the customers takes taxis there, then no extra travel time will be required (i.e., local circulation time is zero). It is considered that taxi customers might attempt to minimize the local circulation time by walking to and then waiting at the roadside where the traffic stream direction is heading to their destination (the local best point) to minimize the travel fare. In our study, the extra travel times to the destination for all possible options to the customers are compared with that of the trip starting from the local best point and evaluate how the prolonged in-vehicle travel time affects the search decisions of the customers.

From the graphical example in Figure 3 assuming that the customer is currently at the roadside of an inner road (i.e., location C), he/she can select of one of the three choices for getting a vacant taxi. Clearly, no walking time is needed for choice (1): waiting at the current position, but choice (2) (walk to and wait at a taxi stand) and choice (3) (waiting at the roadside of a main street where the traffic stream is heading to the passenger's destination) require walking. Considering the necessary circulation time at the local road network, the travel times for choices (1) and (3) are longer than that for choice (2). In this example, the intersection of the inner road network and the main street (i.e., location $\mathrm{M}$ ) is considered the 
local best point for waiting out of the three choices, where requires the minimum travel time to the passenger's destination.

Accordingly, taxi customers are considered to make their choices of hailing taxis at different locations based on three attributes, namely the walking time, the waiting time, and the extra in-vehicle travel time to their destinations. These three attributes were used to generate different hypothetical situations for the taxi customers to decide waiting along the roadside or at taxi stands. For the taxi customers who decided waiting at the roadside of the current position for vacant taxis, the required walking time was obviously equal to zero.

Table 1 tabulates the attributes and their levels for the decisions of taxi customers hailing vacant taxis on streets. All these quantitative attributes were defined at three levels based on the results from the pilot survey. The fractional factorial design method was applied to generate 36 profiles for our choice experiments. The choice sets were randomly divided into 9 sets of questionnaires and distributed to taxi customers.

Table 1. Attributes and levels used for the stated preference survey

\begin{tabular}{|l|l|l|}
\hline \multicolumn{1}{|c|}{ Choices } & \multicolumn{1}{|c|}{ Attributes } & Levels \\
\hline \multirow{2}{*}{$\begin{array}{l}\text { (1) Wait at the roadside } \\
\text { of the current position }\end{array}$} & Waiting time at the current position (min) & $4,6,8$ \\
\cline { 2 - 3 } $\begin{array}{l}\text { (2) Walk to and then } \\
\text { wait at the roadside of a } \\
\text { main street }\end{array}$ & Extra travel time $^{\mathrm{a}}$ to the destination (min) & $0,1,2$ \\
\cline { 2 - 3 } & Walking time $\mathrm{b}^{\mathrm{b}}$ to a main street (min) & $1,3,5$ \\
\cline { 2 - 3 } $\begin{array}{l}\text { (3) Walk to and then } \\
\text { wait at a taxi stand }\end{array}$ & Waiting time at the roadside of a main street (min) & $1,3,5$ \\
\cline { 2 - 3 } & Extra travel time $^{\mathrm{a}}$ to the destination (min) & $0,1,2$ \\
\cline { 2 - 3 } & Walking time $^{\mathrm{b}}$ to a taxi stand (min) & $3,5,7$ \\
\cline { 2 - 3 } & Extra travel time ${ }^{\mathrm{a}}$ to the destination (min) & $0,1,2$ \\
\hline
\end{tabular}

Note: ${ }^{\mathrm{a}}$ Additional time needed for a vacant taxi circulating within a local road network to heading to passenger's destination, compared with the trip starting at the local best point.

${ }^{\mathrm{b}}$ The walking distance in one minute in an urban area is assumed to be $70 \mathrm{~m}$.

Figure 4 shows an example of the choice set in the questionnaire for asking the respondents to select their most preferred option from the three given alternatives in each of the hypothetical games. We invited the respondents to report their most frequent period and region taking taxis in the previous part of the questionnaire as described in Section 2.2. Based on their responses, we requested them to make a choice if they were searching for a vacant taxi in a residential/commercial district in their reported period and region.

Passengers' behavior of hailing vacant taxis (Please $\checkmark$ as appropriate)

Assume that you are now searching for a vacant taxi in a Residential District in the period of time and region you most frequently took a taxi. In the following independent games, which one is the most preferred option for you?

\begin{tabular}{|c|c|c|}
\hline \multirow{3}{*}{ Game 1} & $\begin{array}{l}\text { Wait at the roadside of the current position in } \underline{4 \mathrm{~min}} \text { for a taxi. The taxi travel direction is not } \\
\text { heading to your destination, and hence } \underline{1 \text { extra } \min \text { of travel time is needed for local circulation. }}\end{array}$ & $\square$ \\
\hline & $\begin{array}{l}\text { Walk to a main street in } \underline{\mathbf{1} \text { min }} \text { and } \underline{\text { wait }} \text { for a taxi in } \underline{\mathbf{3} \text { min. }} \text {. The taxi travel direction is heading to } \\
\text { your destination, and hence no extra min of travel time is needed for local circulation. }\end{array}$ & 7 \\
\hline & $\begin{array}{l}\text { Walk to a taxi stand in } \underline{\mathbf{5} \text { min }} \text { and } \underline{\text { wait }} \text { for a taxi in } \underline{\mathbf{2} \text { min }} \text {. The taxi travel direction is not heading } \\
\text { to your destination, and } \underline{\mathbf{2} \text { extra min of travel time is needed for local circulation. }}\end{array}$ & \\
\hline
\end{tabular}

Figure 4 Example of a choice set 
To ensure data quality for model calibration, each interviewed taxi customer was requested to report his/her choice decisions in 4 hypothetical games (2 games for residential districts, and 2 games for commercial districts), and hence 4,968 observations were collected from 1,242 taxi customers in this study.

\section{METHOD}

\subsection{Multinomial Logit Model}

Based on the data collected in the SP questionnaire survey, we adopted the MNL modeling approach at first to depict the behavior of taxi customers in search for vacant taxis on streets. It is commonly used when at least two alternatives exists. The model helps us determine the factors that affect the taxi customers' decisions. The logit model takes the following form [40]:

$$
P_{q}(j)=\frac{\exp \left[U_{q}(j)\right]}{\sum_{m} \exp \left[U_{q}(m)\right]},
$$

where $P_{q}(j)$ is the probability that an individual taxi customer $q$ chooses alterative $j$ to get a taxi. $U_{q}(j)$ is the deterministic utility that captures the factors influencing the decision of taxi customer $q$ to choose $j$. According to Equation (1), the higher utility of a choice implies a higher probability of that choice being selected.

The utility in Equation (1) is modeled as follows:

$$
U_{q}(j)=\beta_{\mathrm{K}} T_{j}^{\mathrm{k}}+\beta_{\mathrm{T}} T_{j}^{\mathrm{t}}+\beta_{\mathrm{L}} T_{j}^{\mathrm{l}}+\beta_{\mathrm{M}} M_{j},
$$

where $T_{j}^{\mathrm{k}}, T_{j}^{\mathrm{t}}, T_{j}^{\mathrm{l}}$, and $M_{j}$ are the walking time, the waiting time, the extra travel time to the destination, and the taxi stand dummy variable, respectively, and $\beta_{\mathrm{K}}, \beta_{\mathrm{T}}, \beta_{\mathrm{L}}$, and $\beta_{\mathrm{M}}$ are their corresponding coefficients. The taxi stand dummy variable was additionally introduced in Equation (2) to determine the general perception of taxi customers, other than the first three attributes, of using taxi stands for meeting vacant taxis. The dummy variable equals one, if the choice is finding taxis at taxi stands. Otherwise, it equals negative one.

It is important to clarify that the attributes in the utility function are indeed the perceived values to a taxi customer for the associated decision of either waiting at a taxi stand or along the roadside for vacant taxis. They were expected to judge the options based on their experience of hailing taxis. We assumed that every taxi customer has the same perception to the utility function attributes in this study. Therefore, the subscript $q$ is omitted in each attribute in Equation (2) for simplicity. Moreover, because of the perception, every taxi customer may not know exactly the value of each attribute and which choice having the actual smallest utility. Hence, a taxi customer does not necessary choose the location with the actual smallest utility.

\subsection{Nested Logit Model}

To account for the potential correlations among the three alternatives, NL models (with two levels: upper and lower nests) are developed and calibrated in the investigation on the alternative similarity. The probability that an individual taxi customer $q$ eventually chooses 
alternative $j \in A_{i}$ to get a taxi is equal to the probability of choosing branch $i \in B$ in the upper nest, $P_{q}(i)$, times the probability of subsequently choosing alternative $j$ in the lower nest after choosing branch $i, P_{q}(j \mid i)$, where $A_{i}$ denotes the set of alternatives under branch $i$ in the lower nest and $B$ denotes the set of branches in the upper nest. Therefore, it can be expressed as:

$$
P_{q}(j)=P_{q}(j \mid i) P_{q}(i) .
$$

The probability of choosing alternative $j$ in the lower nest is calculated using the MNL model:

$$
P_{q}(j \mid i)=\frac{\exp \left[U_{q}(j \mid i)\right]}{\sum_{m \in A_{i}} \exp \left[U_{q}(m \mid i)\right]},
$$

where $U_{q}(j \mid i)$ is the deterministic utility of alternative $j$ if branch $i$ is selected, and is calculated using Equation (2).

The probability of choosing branch $i$ in the upper nest is estimated as:

$$
\begin{gathered}
P_{q}(i)=\frac{\exp \left[\theta_{i} S_{q}(i)\right]}{\sum_{m \in B} \exp \left[\theta_{m} S_{q}(m)\right]}, \text { and } \\
S_{q}(i)=\ln \sum_{n \in A_{i}} \exp \left[U_{q}(n \mid i)\right],
\end{gathered}
$$

where $S_{q}(i)$ is the logsum variable for all alternatives under branch $i . \theta_{i}$ is the associated parameter which captures the correlation between alternatives within branch $i$ and has to be bounded by one and zero. The parameter is predetermined as one if the nest has only one alternative. If the logsum parameter $\theta_{i}=1$ for all branches, the NL model is equivalent to the MNL model.

In this study, one possible nested structure is to group "walk to and then wait at the roadside of a main street" and "walk to and then wait at a taxi stand" together. This nest can be referred to as "prefer walking to search for a vacant taxi". Another possibility is to group "wait at the roadside of the current position" and "walk to and then wait at the roadside of a main street" together, and refer to the group as "not prefer waiting at a taxi stand”. The MNL model can be considered a special case of an NL model, when the three alternatives are found to be uncorrelated and they can be treated as three parallel choices in the same nest. The nested structures for the three models are shown in Figure 5. All of these nested structures are tested in this study. 


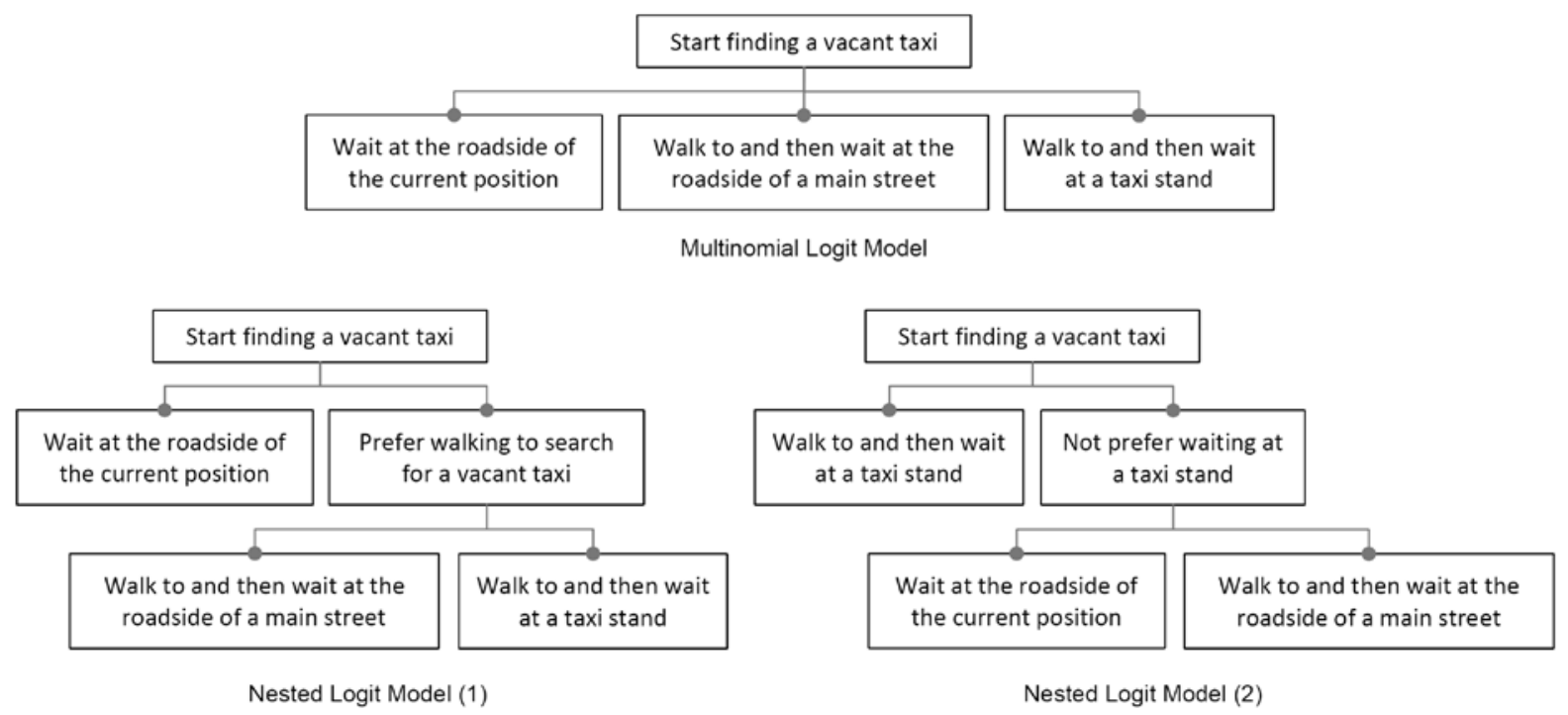

Figure 5. All possible nested structures

\subsection{Model Calibration}

All the variable coefficients in the MNL and NL models are calibrated under the maximum log-likelihood principle by considering the following objective function:

$$
\text { Maximize } \mathrm{LL}=\sum_{j} \ln P_{q}(j),
$$

where LL is the log-likelihood of the MNL/NL model, in which $P_{q}(j)$ is the calculated probability of selecting alternative $j$ of an individual customer $q$ as calculated by Equation (1) for the MNL model or Equation (3) for the NL models.

\subsection{Model Comparison and Market Segmentation Test}

To select the best model structure from the three proposed models, the likelihood ratio test, may also refer to as Watson and Westin pooling test [41], are conducted to compare the goodness-to-fit of the models. The test is based on the log-likelihood ratio LR, which is calculated as:

$$
\mathrm{LR}=-2\left(L_{\mathrm{R}}-L_{\mathrm{U}}\right) \text {, }
$$

where $L_{R}$ denotes the log likelihood for the restricted MNL model and $L_{U}$ denotes the log likelihood for the unrestricted NL model. The null hypothesis that there is no intervention between the two models is rejected, as the test statistic exceeds the threshold value that is specified for the chi-squared distribution at the chosen level of significance. The degree of freedom is calculated as the difference between the numbers of variables of the two models.

In addition, to evaluate the variations in the search behaviors of taxi customers in different districts, periods, and regions, the above likelihood ratio test can also be employed for market segmentation analysis. In this case, $L_{\mathrm{R}}$ represents the log likelihood for the base model that is calibrated for the combined dataset with all market segments (either in terms of districts, periods or regions, but not both simultaneously), and $L_{U}$ represents the sum of the $\log$ likelihoods of the sub-models calibrated for different market segments. Similarly, the null hypothesis that there is no intervention in market segmentation is rejected, as the test statistic exceeds the threshold value that is specified for the chi-squared distribution at the chosen 
level of significance. The degree of freedom is calculated as the difference between the number of variables of the base model and the sum of the number of individual sub-models.

\section{RESULTS AND DISCUSSION}

\subsection{Results of the base models and model comparison}

NLOGIT was adopted for model calibration in this study. NLOGIT is an extension of the econometric and statistical software package LIMDEP [42], which uses the maximum likelihood estimation method to determine the coefficient of each variable in the discrete choice models based on respondents' selections among a set of competing alternatives. Various model specifications were examined. The coefficient of each variable of the standard MNL model and the best nested logit model were determined and are tabulated in Table 2 . All the coefficients are found to be significant at the $1 \%$ level. Hence, it is proved that the walking time, the waiting time, and the extra travel time to the destination significantly affect the decisions of taxi customers hailing vacant taxis on streets.

Table 2. Results of the base models and the likelihood ratio test

a. Results of the multinomial logit and nested logit models

\begin{tabular}{|l|c|c|}
\hline \multirow{2}{*}{\multicolumn{1}{|c|}{ Explanatory variables }} & \multicolumn{2}{c|}{ Coefficients $^{\mathrm{a}}$ [t-statistics] } \\
\cline { 2 - 3 } & Multinomial Logit Model & Nested Logit Model (1) \\
\hline Walking time & $-0.24[-21.5]$ & $-0.32[-18.7]$ \\
\hline Waiting time & $-0.34[-29.1]$ & $-0.30[-24.1]$ \\
\hline Extra travel time to the destination & $-0.55[-20.5]$ & $-0.58[-20.7]$ \\
\hline Taxi stand dummy variable & $-0.26[-10.5]$ & $-0.20[-7.2]$ \\
\hline Logsum variable & -- & $0.75[21.3]$ \\
\hline
\end{tabular}

b. Results of the likelihood ratio test

\begin{tabular}{|c|c|c|}
\hline \multicolumn{2}{|c|}{ Measures/Conclusions } & Results \\
\hline \multirow{2}{*}{$\begin{array}{l}\text { Log } \\
\text { likelihood }\end{array}$} & The multinomial logit model & $-4,662.0$ \\
\hline & The nested logit model (1) & $-4,641.2$ \\
\hline \multicolumn{2}{|c|}{ Likelihood ratio (LR) } & 41.6 \\
\hline \multicolumn{2}{|c|}{ Chi-square critical value $^{\mathrm{c}}$} & 6.6 \\
\hline \multicolumn{2}{|c|}{ Conclusion of the hypothesis test $^{\mathrm{d}}$} & Reject \\
\hline
\end{tabular}

Note: ${ }^{a}$ All parameters are significant at the $1 \%$ level.

${ }^{b}$ The values in a pair of brackets represent the t-statistics of the explanatory variables.

${ }^{c}$ The chi-square critical value when the degree of freedom is 1 and the significance level is 0.01 .

${ }^{\mathrm{d}}$ The null hypothesis test at the $99 \%$ confidence interval.

According to Table 2, all the three time related attributes in both the MNL and NL models have negative coefficients. It implies that the taxi customers searched for vacant taxis on streets following the time minimization rule. They prefer the hailing location with lower walking time, waiting time, and extra travel time to the destination. The coefficient of the taxi stand dummy variable is found to be negative as well. It illustrates the average perception of taxi customers that they prefer waiting along the roadside to waiting at taxi stands to hail 
vacant taxis. The negative coefficient also agrees with the results in Figure 2 that more people they prefer waiting along the roadside to waiting at taxi stands to hail vacant taxis. Overall, the sign of each coefficient is logical in term of representing taxi customers' search decisions and meet our expectations.

Specifically for the NL models, the parameter associated with the logsum variable is additionally calibrated. It is one of the selection criteria to choose the best nested structure. The logsum parameter for the NL model (1) is 0.75 with a standard deviation of 0.04 . The upper and lower bounds of estimation at the 99\% confidence interval are 0.63 and 0.87 respectively. It provides statistical evidence that the logsum parameter is between zero and one, and implies that the correlation between "walk to and then wait at the roadside of a main street" and "walk to and then wait at a taxi stand" is statistically significant. On the other hand, the logsum parameter for the NL model (2) is 1.19. It shows that the model structure is invalid and the correlation between "wait at the roadside of the current position" and "walk to and then wait at the roadside of a main street" is arguable.

Table 2 also shows the log-likelihood values of the two models, which are used to calculate the log-likelihood ratio. Given that the degree of freedom in 1 , and the chi-square critical value at the $1 \%$ level of significant is 6.6 , which is significantly lower than the loglikelihood ratio of 41.6. Therefore, the null hypothesis that there is no intervention between the two models is rejected accordingly. Since the NL model (1) gives a higher maximum loglikelihood value than that of the MNL model. The likelihood ratio test result demonstrates that the nested logit model (1) is superior to the standard MNL model, and better fits the survey data.

In addition, the differences of the calibrated coefficients of all time related attributes in the finalized NL model can tell us the taxi customers' perceptions on different time consumptions. Theoretically, for every one minute spent on walking, waiting, or in-vehicle travel should result in the same amount of disutility to the model function under the time minimization principle. However, the magnitude of the coefficients illustrates that the taxi customers preferred waiting to walking, which has the smallest absolute value to the others. As described in Figure 2, about 24\% of respondents took taxis because of carrying heavy luggage. They would prefer searching for vacant taxis in a shorter walking distance. Reversely, prolonging the in-vehicle travel time was the least preferable, because it not only increased the overall time spent in traveling to the destination, but also resulted in a higher travel fare charge to them as well.

\subsection{Model results for different market segments}

Table 3 presents the results of the market segmentation analysis. The market segments were defined by two types of districts (residential and commercial districts), two periods (day time and night time), and three regions (Hong Kong Island, Kowloon Peninsula, and the New Territories). Table 3 also gives the log likelihood values of the base model of the finalized NL model and the sum of the individual models for different market segments, both of which were used to calculate the log likelihood ratios. The degree of freedom for each market segmentation analysis is determined as the difference between the sum of the number of parameters associated with each segment and the number of parameters of the combined model.

For the district and region segmentation analysis, the log likelihood ratios are significantly higher than the associated chi-square critical value at the $1 \%$ significant level. Therefore, the null hypotheses that there are no interventions in districts and regions are rejected. As a result, we conclude that they vary from the others and cannot be pooled together. Reversely, the log 
likelihood ratio for the period segmentation analysis is lower than the chi-square critical value. The null hypothesis that there are no interventions between during different periods of taking taxis is not rejected. The implication is that no significant difference is found between the customers' taxi-search behaviors during different periods.

Table 3. Model results for different market segments

a. Results of the market segmentation analysis

\begin{tabular}{|l|c|c|c|c|c|c|}
\hline \multicolumn{1}{|c|}{ Market segments } & $L_{\mathrm{R}}$ & $L_{\mathrm{U}}$ & $\begin{array}{c}\text { Degree of } \\
\text { freedom }\end{array}$ & LR & $\begin{array}{c}\text { Critical } \\
\text { value }\end{array}$ & Conclusion \\
\hline District (land use) & $-4,641.2$ & $-4,623.9$ & 5 & 34.6 & $15.1^{\mathrm{a}}$ & Reject \\
\hline Period & $-5,035.0$ & $-5,030.9$ & 5 & 8.2 & $11.1^{\mathrm{b}}$ & Do not reject \\
\hline Region & $-5,149.6$ & $-5,140.0$ & 10 & 19.2 & $18.3^{\mathrm{b}}$ & Reject \\
\hline
\end{tabular}

b. Results of the sub-models

\begin{tabular}{|c|c|c|c|c|}
\hline \multirow{2}{*}{ Explanatory variables } & \multicolumn{4}{|c|}{ Coefficients $^{\mathrm{c}}$ [t-statistics] $^{\mathrm{d}}$} \\
\hline & \multicolumn{2}{|c|}{ Residential district } & \multicolumn{2}{|c|}{ Commercial district } \\
\hline Walking time & \multicolumn{2}{|c|}{$-0.32[-12.5]$} & \multicolumn{2}{|c|}{$-0.32[-13.9]$} \\
\hline Waiting time & \multicolumn{2}{|c|}{$-0.30[-16.8]$} & \multicolumn{2}{|c|}{$-0.31[-17.4]$} \\
\hline Extra travel time to the destination & \multicolumn{2}{|c|}{$-0.68[-16.8]$} & \multicolumn{2}{|c|}{$-0.48[-12.2]$} \\
\hline Taxi stand dummy variable & \multicolumn{2}{|c|}{$-0.28[-7.1]$} & \multicolumn{2}{|c|}{$-0.12[-3.1]$} \\
\hline Logsum variable & \multicolumn{2}{|c|}{$0.75[15.5]$} & \multicolumn{2}{|c|}{$0.76[14.6]$} \\
\hline Explanatory variables & $\begin{array}{l}\text { Hong Kong } \\
\text { Island }\end{array}$ & & & $\begin{array}{c}\text { New } \\
\text { Territories }\end{array}$ \\
\hline Walking time & $-0.33[-10.9]$ & -0.3 & $-11.5]$ & $-0.31[-11.1]$ \\
\hline Waiting time & $-0.31[-14.8]$ & -0.2 & $14.4]$ & $-0.28[-13.1]$ \\
\hline Extra travel time to the destination & $-0.50[-10.8]$ & -0. & -13.7] & $-0.62[-13.2]$ \\
\hline Taxi stand dummy variable & $-0.24[-5.2]$ & & $-3.6]$ & $-0.16[-3.5]$ \\
\hline Logsum variable & $0.74[12.4]$ & 0. & 12.8] & $0.73[12.0]$ \\
\hline
\end{tabular}

Note: ${ }^{a}$ Chi-square critical value at the $1 \%$ significant level.

${ }^{\mathrm{b}}$ Chi-square critical value at the $5 \%$ significant level.

${ }^{\mathrm{c}}$ All the parameters are significant at the $1 \%$ level.

${ }^{\mathrm{d}}$ The values in brackets represent the t-statistics of the explanatory variables.

Table 3 also presents the detailed results of each of the sub-models. All coefficients are negative and agree with our expectation. It shows the differences in customers' taxi-search behaviors in various districts and regions.

For the sub-models of residential and commercial districts, it is observed that the changes in the taxi customers' perceptions on the walking time and the waiting time are minimal across districts. However, the absolute value of the coefficients of the extra travel time to the destination and taxi stand dummy variable are obviously smaller in the commercial district sub-model. It suggests that customers in commercial districts had less concern on these attributes when hailing vacant taxis on streets. It can be interpreted that commercial districts typically had a higher passenger demand, whereas the competition between taxi customers in search of vacant taxis was too high. The taxi customers comparatively did not mind paying extra travel fare and going to taxi stands, as long as they could catch one in a shorter time.

Table 3 also provides an idea of the variations in the search strategies of taxi customers in different regions. As reflected by the coefficients of all attributes in the model results, taxi 
customers in Kowloon Peninsula and the New Territories had a relatively similar vacant taxi search strategy, whereas Hong Kong Island taxi customers made their decisions substantially different. In particular, they tended to spend the shortest time to get in a vacant taxi and comparatively had a less concern on the extra travel time to the destination. Thus, they had a stronger resistance to wait at taxi stands. It may be because the number of taxi stands established in Hong Kong Island were insufficient and some of these taxi stands were located at remote areas and not convenient for the taxi customers there.

\subsection{Discussion: policy implications and recommendations}

The magnitude of the coefficient of the taxi stand dummy variable reflects the conceptual degree of dissatisfaction of taxi customers about walking to and waiting at taxi stands for getting taxis. A negative coefficient of this dummy variable with a small absolute value shows that taxi customers had a tendency of not going to taxi stands but the associated resistance was weak. According to Equation (2), the disutility of the perception on using taxi stands is found to be similar to that of spending one more minute in taxi-search. Based on the magnitudes of all other calibrated coefficients, we can conclude that slightly adjusting the value of one of the other three attributes will significantly affect the decision choices of taxi customers in search of vacant taxis. Hence, we can use each of these sub-models to examine the effect of the policies that affect the value of one of the three attributes. For example, introducing more taxi stands reduces the required walking time to nearby taxi stands because a shorter walking time increases the probability of taxi customers selecting taxi stands as their choice in searching for taxis according to the model results.

Because the extra travel time has the highest disutility to the model function, taxi stands located at the local best point for taxi customers is highly recommended. However, taxi passengers had different destinations. The location of local best point is hard to be identified. To dissolve this problem, the origin-destination pattern of taxi passengers is worth to be studied before setting up a taxi stand in an urban area. If the ways to their destinations are found to be highly dispersed, taxi stands are then suggested to be established near junctions (with a condition of causing low disturbance to local traffic) allowing all turning movements to different directions, instead of setting up in the middle of a long road segment, for minimizing the extra travel time required for local circulation.

As suggested by the interviewed taxi customers, more direction signs elected on streets are also recommended for guiding people to the nearby taxi stands. Since more than $20 \%$ of interviewees took taxis at unfamiliar areas, and certainly they could not find a taxi stand in the surrounding without a guidance system. Clear and appropriate direction signs can promote more people walking to and then waiting at taxi stands instead of hailing along the roadside.

As found in the previous study of Wong et al. [30], because of higher passenger demand at commercial districts, many vacant taxis were attracted to these districts and circulate on the road network for customers, which worsened the local traffic congestion. The search strategies of drivers were also found to depend on the type of districts. On the other hand, based on the sub-model results, we found that people in commercial districts actually did not have a strong negative perception to wait at taxi stands, and that the search strategy of customers also depends on the type of districts. Hence, we believe that encouraging both taxi customers and taxi drivers meeting at taxi stands in commercial districts has a high potential to minimize their search time on streets and thus enhance the overall meeting efficiency. Taxi policies should be implemented towards this direction. 
The region segmentation analysis shows that the sub-model of Hong Kong Island has higher absolute values of the walking time coefficient and the taxi stand dummy variable coefficient than the other two sub-models. Hence, introducing additional taxi stands in Hong Kong Island will be less effective than that in Kowloon Peninsula and the New Territories since introducing more taxi stands results in a lower walking time to the nearest taxi stand.

As implied by the calibrated model, attracting more vacant taxis arriving at taxi stands is an effective way to enhance the usage of taxi stands by taxi customers (because it leads to a shorter waiting time). More vacant taxis waiting at taxi stands for customers form a virtuous cycle to encourage more taxi customers going these locations. Therefore, how vacant taxi drivers will react after providing more taxi stands indeed is a crucial component to evaluate the consequences of the preceding, proposed transportation policies. Actually, Wong et al. [30] performed a study in this direction by developing a model to depict and examine the behavior of taxi drivers traveling towards taxi stands. Although the focus of their paper is different from our paper, their findings supported our policy recommendation on setting up more taxi stands to increase the usage in general. They also found that setting up additional taxis in Hong Kong Island is less effective than in Kowloon Peninsula and the New Territories.

Based on the findings of this paper and Wong et al. [30], we can conclude that both taxi customers and taxi drivers did not have a strong resistance of using taxi stands, and they made selections based on the real-time condition. This implies that if the real-time information at or around taxi stands (e.g., the number of vacant taxis at taxi stands and the congestion level around taxi stands) was provided to both taxi drivers and customers, then the usage of taxi stands by them could be further improved. Hence, implementing taxi policies related to providing real-time taxi stand information is also recommended.

\section{CONCLUSION}

This study analyzes the preferences of taxi customers for hailing vacant taxis on streets. A stated preference survey was conducted to interview 1,242 respondents to capture their choices in different hypothetical scenarios. These respondents were either taxi customers who were waiting at taxi stands or pedestrians on streets who had experiences of taking taxis recently. 4,968 observations were collected and used to develop and calibrate the discrete choice models for policy analysis.

The calibrated models show that the walking time, the waiting time, the extra travel time to the destination, and the preference of a taxi customer for walking to and then waiting at taxi stands are found to be significant factors that influence their decisions on selecting locations of finding vacant taxis on streets. In particular, taxi customers generally searched for vacant taxis based on the time minimization concept and they generally preferred searching for taxis along the roadside to traveling towards a taxi stand. However, there are perception errors on the walking, waiting, and in-vehicle travel times among taxi customers and some negative perception on getting taxis at taxi stands. Hence, a taxi customer does not necessary choose the location with the actual smallest utility.

The coefficients of the variables are firstly estimated using a standard MNL model. In order to account for the potential correlations among the three alternatives, two NL models are calibrated in the investigation. The results of likelihood ratio test lead to a conclusion that a NL model is recommended over the standard MNL model to describe the search behavior of taxi customers. The results also show that there is a correlation between the alternative 
"walk to and then wait at the roadside of a main street" and the alternative "walk to and then wait at a taxi stand".

The market segmentation analysis demonstrates the variations in the preferences of taxi customers for searching for taxis in different districts and regions in Hong Kong during various periods. The model results illustrate that the taxi-search strategies of taxi customers were substantially different in various districts and regions but there is insufficient evidence to demonstrate that the search strategies were different during various periods.

This paper also discusses potential taxi policy implications on enhancing the utilization rates of taxi stands and on introducing additional taxi stands in different districts and regions. Our model helps predicting how taxi customers search on streets for a vacant taxi under different taxi stand arrangements. This model can be served as one of the sub-models in a two-stage micro-simulation model, which integrates with the model of Wong et al. [30] about modeling the vacant taxi drivers' decisions to taxi stands for customers. The first stage model is to depict the vacant taxi customer-search decisions patterns on streets and the second stage model is to estimate the customers' movements on streets for finding vacant taxis. These two sub-models are developed to influence each other, and an iterative solution procedure is necessary to solve for a convergent solution. The average waiting times for both taxi drivers and customers can be estimated under different taxi stand arrangements. Thus, an optimal solution with the least waiting times can be determined to maximize the meeting efficiency between taxi drivers and customers.

In addition, we suggest the following research directions for future study to improve the model findings: (1) collecting the revealed preference choices of taxi customers on the three search strategies and applying our model to confirm the model accuracy in predicting the market share to validate the model findings; (2) extending our model to facilitate the possible choices of taxi customers of hailing different types of taxis with different charging rates (if their origins and destinations are within an overlapping operational area); and (3) modeling the possible decision changes of taxi customers in selecting other transport modes on the way of searching vacant taxis on streets and capturing the perceived vacant taxi proportion on streets in the model.

\section{ACKNOWLEDGEMENTS}

This research was jointly supported by two grants (201111159056 and 201209160014) from the University Research Committee, a Research Postgraduate Studentship, and a Faculty of Engineering Top-up Grant from the University of Hong Kong, a grant from National Natural Science Foundation of China, China (71271183), and a grant from the Research Grants Council of the Hong Kong Special Administrative Region, China (HKU 716913E). The authors also wish to thank Jamie Kit Man CHOI, a student of Civil Engineering Department, The University of Hong Kong for her assistance for data collection and analysis. The authors are also grateful to the constructive comments from the five reviewers.

\section{REFERENCES}

1. Austin D, Zegras PC. Taxicabs as public transportation in Boston, Massachusetts. Transportation Research Record: Journal of the Transportation Research Board 2012, 2277: 65-74. 
2. King DA, Peters JR, Daus MW. Taxicabs for improved urban mobility: Are we missing an opportunity? Proceedings of the 91st Transportation Research Board Annual Meeting, Washington, D.C. United States 2012.

3. Lee DH, Wang H, Cheu RL, Teo SH. Taxi dispatch system based on current demands and real-time traffic conditions. Transportation Research Record: Journal of the Transportation Research Board 2004, 1882:193-200.

4. Lee KT, Lin DJ, Wu PJ. Planning and design of a taxipooling dispatching system. Transportation Research Record: Journal of the Transportation Research Board 2005, 1903:86-95.

5. Tao CC. Dynamic taxi-sharing service using intelligent transportation system technologies. Proceedings of International Conference on Wireless Communications, Networking and Mobile Computing 2007:3209-3212.

6. Chang JSK, Wu CH, Lin CH. Optimization of fleet size for GPS taxi. Proceedings of the 91st Transportation Research Board Annual Meeting, Washington, D.C. United States 2012.

7. Conway A, Kamga C, Yazici A, Singhal A. Challenges in managing centralized taxi dispatching at high-volume airports: Case study of John F. Kennedy International Airport. Transportation Research Record: Journal of the Transportation Research Board 2012, 2300:83-90.

8. da Costa DCT, de Neufville R. Designing efficient taxi pickup operations at airports. Transportation Research Record: Journal of the Transportation Research Board 2012, 2300:91-99.

9. Manski CF, Wright JD. Nature of equilibrium in the market for taxi services. Transportation Research Record: Journal of the Transportation Research Board 1976, 619: 296-306.

10. Arnott R. Taxi travel should be subsidized. Journal of Urban Economics 1996, 40:316333.

11. Schaller B. Issues in fare policy: Case of the New York taxi industry. Transportation Research Record: Journal of the Transportation Research Board 1998, 1618:139-142.

12. Fernández JE, de Cea J, Briones J. A diagrammatic analysis of the market for cruising taxis. Transportation Research Part E 2006, 42:498-526.

13. Loo BPY, Leung BSY, Wong SC, Yang H. Taxi license premiums in Hong Kong: can their fluctuations be explained by taxi as a mode of public transport? International Journal of Sustainable Transportation 2007, 1:249-266.

14. Yang H, Wong SC. A network model of urban taxi services. Transportation Research Part B 1998, 32:235-246.

15. Wong KI, Wong SC, Yang H. Modeling urban taxi services in congested road networks with elastic demand. Transportation Research Part B 2001, 35:819-842.

16. Wong KI, Wong SC, Yang H, Wu JH. Modeling urban taxi services with multiple user classes and vehicle modes. Transportation Research Part B 2008, 42:985-1007.

17. Kim H, Oh JS, Jayakrishnan R. Effect of taxi information system on efficiency and quality of taxi services. Transportation Research Record: Journal of the Transportation Research Board 2005, 1903:96-104.

18. Yang H, Fung CS, Wong KI, Wong SC. Nonlinear pricing of taxi services. Transportation Research Part A 2010, 44:337-348.

19. Yang T, Yang H, Wong SC, Sze NN. Returns to scale in the production of taxi services: an empirical analysis. Transportmetrica A 2014, 10: 775-790. 
20. Hu X, Gao S, Chiu YC, Lin DY. Modeling routing behavior for vacant taxicabs in urban traffic networks. Transportation Research Record: Journal of the Transportation Research Board 2012, 2284:81-88.

21. Wong RCP, Szeto WY, Wong SC. A cell-based logit-opportunity taxi customer-search model. Transportation Research Part C 2014, 48:84-96.

22. Wong SC, Yang H. Network model of urban taxi services: improved algorithm. Transportation Research Record: Journal of the Transportation Research Board 1998, 1623:27-30.

23. Wong KI, Wong SC, Yang H, Tong CO. A sensitivity-based solution algorithm for the network model of urban taxi services. In: Taylor, M.A.P. (ed.), Proceedings of the 15th International Symposium on Transportation and Traffic Theory. Amsterdam: Elsevier Science 2002:23-42.

24. Wong KI, Wong SC, Yang H, Tong CO. The effect of perceived profitability on the level of taxi service in remote areas. Journal of the Eastern Asia Society for Transportation Studies 2003, 5:79-94.

25. Sirisoma RMNT, Wong SC, Lam WHK, Wang D, Yang H, Zhang P. Empirical evidence for taxi customer-search model in Hong Kong. Proceedings of the Institution of Civil Engineers, Transport 2010, 163:203-210.

26. Wong RCP, Szeto WY, Wong SC, Yang H. Modeling multi-period customer-searching behaviour of taxi drivers. Transportmetrica B 2014, 2:40-59.

27. Szeto WY, Wong RCP, Wong SC, Yang H. A time-dependent logit-based taxi customersearch model. International Journal of Urban Sciences 2013, 17:184-198.

28. Wong RCP, Szeto WY, Wong SC. Sequential logit approach to modeling the customersearch decisions of taxi drivers. Asian Transport Studies 2015, in press.

29. Kitamura R, Yoshii T. Rationality and heterogeneity in taxi driver decisions: Application of a stochastic-process model of taxi behavior. In Mahmassani, H. (ed.) Transportation and Traffic Theory: Flow, Dynamics, and Human Interaction. Elsevier Science 2005, 609-628.

30. Wong RCP, Szeto WY, Wong SC. Bi-level decisions of vacant taxi drivers traveling towards taxi stands in customer-search: modeling methodology and policy implications. Transport Policy 2014, 33:73-81.

31. Wong KI, Wong SC, Bell MGH, Yang H. Modeling the bilateral micro-searching behavior for urban taxi services using the absorbing Markov chain approach. Journal of Advanced Transportation 2005, 39:81-104.

32. Yang H, Leung CWY, Wong SC, Bell MGH. Equilibria of bilateral taxi-customer searching and meeting on networks. Transportation Research Part B 2010, 44:10671083.

33. Yang H, Yang T. Equilibrium properties of taxi markets with search frictions. Transportation Research Part B 2011, 45:619-742.

34. Yang T, Yang H, Wong SC. Taxi services with search frictions and congestion externalities. Journal of Advanced Transportation 2012, DOI: 10.1002/atr.1210.

35. Hensher DA. A nested logit stated choice analysis of pricing options. Journal of Advanced Transportation 1998, 32:130-151.

36. Forinash CV, Koppelman FS. Application and interpretation of nested logit models of intercity mode choice. Transportation Research Record: Journal of the Transportation Research Board 1993, 1413:98-106.

37. Lo HK, Yip CW, Wan QK. Modeling competitive multi-modal transit services: a nested logit approach. Transportation Research Part C 2004, 12:251-272. 
38. Lee BHY, Waddell P. Residential mobility and location choice: a nested logit model with sampling of alternatives. Transportation 2010, 37:587-601.

39. Hunt JD, Teply S. A nested logit model of parking location choice. Transportation Research Part B 1993, 27:253-265.

40. McFadden D. Conditional logit analysis of qualitative choice behavior. In: Zarembka, $P$. (ed.), Frontiers in Econometrics. New York: Academic Press 1974, 105-142.

41. Watson PL, Westin RB. Transferability of disaggregate mode choice models. Regional Science and Urban Economics 1975, 5:227-249.

42. Greene WH. NLOGIT Version 4.0: Reference Guide. Econometric Software, Plainview, NY, 2007. 